\title{
Measurement of temperature during simple dynamic shear
}

\author{
by W.K. Nowacki, S.P. Gadaj, E.A. Pieczyska
}

Center of Mechanics and Information Technology, Institute of Fundamental Technological Research PAS, Swietokrzyska 21, 00-049 Warsaw, Poland, e-mail: wnowacki@ippt.gov.pl

\begin{abstract}
This paper presents an application of infrared thermography to investigate the dynamic simple shear of sheets at high strain rates. The methodology of the simple shear deformation are described. Two methods of temperature measurements on the basis of infrared radiation detection were used in order to satisfy the conditions of dynamic investigation. The temperature accompanying the dynamic simple shear has been evaluated.
\end{abstract}

\section{Introduction}

Deformation processes always modify the temperature fields in materials. The temperature variation (which is sensitive to the rate of deformation) is particularly significant for dynamic deformations. In the case of quasi-static deformations, the temperature field due to plastic deformation can be measured by a thermography camera and even the shear macro-bands can be observed [1]. With this technique, one can also evaluate the stored energy due to simple shear, especially when the deformation is large enough.

However, the dynamic deformation at high strain rates requires satisfying the conditions, which make the temperature measurements rather complicated. The specimen is deformed for a period, which is too short to observe the accompanying increase of temperature as a thermography image. We can measure only the mean temperature of two shear zones of the specimen in the minimum time period of $10 \mathrm{~ms}$. The mean maximal increase of the temperature of the specimen of ES steel, according to the French standards, subjected to simple shear at the strain rate $10^{3} \mathrm{~s}^{-1}$, was $60 \mathrm{~K}$. The specimen dimensions were: length $I_{o}=20 \mathrm{~mm}$, width $a_{o}=3 \mathrm{~mm}$ and thickness $d=0.8 \mathrm{~mm}$. The corresponding deformation maximum of the specimen was about $75 \%$.

The measuring system (thermography camera) has no contact with the specimen. This is an important advantage in dynamic tests.

\section{Description of the dynamic simple shear test, equations and results}

Tests in simple shear are important for the experimental investigation of the constitutive relations. Such tests are supplementary to other tests performed in tension, torsion as well as in compression. A simple shear test is particularly attractive, since application of this loading path can cause large strains without the occurrence of plastic instability. In this paper the results of dynamic plane simple shear tests are discussed. The scheme of the standard Split- Hopkinson Pressure Bar (SHPB) apparatus is shown in Fig.1. Use is made of a new double-shear device (Fig. 2) in which loading and displacement are controlled in compression [2]. The role of the special device is to transform the compression into a simple shear. The idea of such transformation is shown in Fig. 2.

The measurement of dynamic deformation at high strain rates in the case of simple shear of the metal sheets is more complicated than in the quasistatic loading. In the case of dynamic deformation with the SHPB, the determination of the relative displacement $\Delta \mathrm{I}$ of the external and internal parts of the shear device is not a quite simple matter. It is assumed that both bars are elastic and displacements $\delta_{1}(t)$ and $\delta_{2}(t)$ of two faces of the shear device can be determined from the measured elastic waves $\varepsilon_{i}, \varepsilon_{t}$ and $\varepsilon_{r}$ : 


$$
\delta_{1}(t)=c_{0} \int_{0}^{t}\left[\varepsilon_{i}(\tau)-\varepsilon_{r}(\tau)\right] d \tau, \quad \delta_{2}(t)=c_{0} \int_{0}^{t} \varepsilon_{t}(\tau) d \tau,
$$

where $c_{o}$ is the longitudinal wave speed in the bars.

The mean shear strain $\bar{\gamma}^{*}$ corresponds to the relative displacement

$$
\bar{\gamma}^{*}=\frac{\Delta l}{a_{o}}=\frac{1}{a_{o}}\left[\delta_{1}(t)-\delta_{2}(t)\right]
$$

The mean shear strain $\bar{\sigma}_{12}$ can be calculated from

$$
\bar{\sigma}_{12}=\left[F_{1}(t)+F_{2}(t)\right] / 4 A_{s}
$$

where $F_{1}(t)$ and $F_{2}(t)$ are forces acting on two ends of the shear device, they can be expressed as functions of $\varepsilon_{i}(t), \varepsilon_{r}(t)$ and $\varepsilon_{t}(t)$ using Hooke's law. The mean shear strain is given by

$$
\sigma_{12}=\frac{1}{4} E_{o} \frac{A_{H}}{A_{s}}\left[\varepsilon_{i}(t)+\varepsilon_{r}(t)+\varepsilon_{t i}(t)\right]
$$

where $E_{o}$ is Young's modulus and $A_{H}$ is the cross section area of Hopkinson bars. It must be kept in mind that all those relations have been derived using the elementary wave theory of longitudinal elastic waves.

We obtain directly the hardening function $\sigma_{12}(\gamma)$ of the examined specimen in simple plane shear. Using this method, we can determine the surface $f\left(\sigma_{12}, \gamma, \dot{\gamma}\right)=0$ if we assume that the time of wave transition through the specimen is very small compared to the total time of loading by the first pulse. A result of multiple reflections of the loading waves in the specimen is the uniform distribution of strains and stresses in the whole gauge section.

An example of stress-strain relations obtained during dynamic deformation with the SHPB is shown in Figure 3.

The highest strain rate in the specimen can be obtained using only one bar of the SHPB system. We use the transmitting bar only (tube) and the shearing device is placed in front of this bar. The flat projectile strikes directly the double shear specimen placed in the device on the front of the transmitting bar, as shown in Fig. 4. We have to register only the transmitted impulse $\varepsilon_{t}$ and the velocity of the projectile.

The mean shear strain and the mean strain rate are [3]

$$
\bar{\gamma}(t)=\frac{1}{a_{0}}\left[v_{0} t-c_{o} \int_{0}^{t} \varepsilon_{t}(\tau) d \tau\right], \quad \dot{\bar{\gamma}}(t)=\frac{1}{a_{0}}\left[v_{o}-c_{o} \varepsilon_{t}(t)\right]
$$

The shear stress can be expressed as

$$
\bar{\sigma}_{12}(t)=\frac{S_{T}}{2 S_{E}} E_{o} \varepsilon_{t}(t)
$$

where $E_{o}$ is the Young modulus of the tube, $S_{T}=\pi\left(d_{2}^{2}-d_{1}^{2}\right) / 4, d_{1}$ and $d_{2}$ are the internal and external diameters of the tube, respectively. Thus, the shear stress in the doubleshear specimen is proportional to the current signal of the transmitted longitudinal wave $\varepsilon_{t}$.

An example of stress-strain relations obtained from the dynamic deformation during direct impact is shown in Fig. 5.

\section{Temperature measurements}

The method of temperature measurements on the basis of the infrared radiation detection was modified in order to satisfy the conditions of dynamic simple shear. In this order the temperature variation was determined by analysing the amplified detector signal produced by the infrared radiation emitted from the specimen surface points which lie on a 
line parallel to the axis $x_{1}$ (see Fig. $2 b$ ), i.e. by analysing the signal of the thermography image line corresponding to the line in the middle of one of the shear zones in the specimen [4]. By stopping one of the rotating prisms in the thermography camera we can reduce the recording time of the image line down to $625 \mathrm{~ns}$. The thermal sensitivity of the camera is better than $0.1 \mathrm{~K}$. When one of the prisms is stopped, the temperature stabilization system in the thermography camera does not work. Therefore an indicator of constant temperature was placed in the observation space of the camera. The temperature of this indicator should be known, it determines a reference level. By sweeping the camera from the left to the right it is possible to take records of two or more time sequences of the radiation power at the shear zone (see Fig. 6).

Our experiments were carried out in order to measure the temperature of the ES steel specimen in the case of simple shear at a strain rate of about $10^{3} \mathrm{~s}^{-1}$. Successive sequences start every $625 \mu \mathrm{s}$. We find that the temperature increases with successive sequences. In the paper [4] it is shown that maximum heating of the shear zone of construction steel occurs in the fourth time sequences. In this paper a calibration of temperature measuring system is also described.

In addition we used a thermography camera with an image rate of $750 \mathrm{~s}^{-1}$ to estimate changes of temperature during dynamic simple shear. An example of such changes obtained during dynamic tests by using this method is shown in Fig.7. The time for one thermography image is too long to measure the temperature changes during the shear test. However, we can estimate temperature changes after this process, during the next sequences of the wave signals. Visual observation of the temperature field distribution during dynamic simple shear allowed also to study how the process of simple shear evolves in the shear zone.

\section{Conclusion}

In the case of a thin sheet, the proposed method is the only known test providing homogeneous stress and strain fields in both dynamic and static tests. They can be used to verify the constitutive relations. The simple shear test is particularly attractive, since the application of this type of loading path can result in large strains without the occurrence of a plastic instability. The advantages of the method in the case of static deformation were discussed previously $[2,3,5]$. The thermo-mechanical coupling formulas have been presented in [1] and [6]. The experimental data of temperature increments received during dynamic simple shear test confirm the worked out theory.

\section{Acknowledgments}

This work has been partially supported by the Polish State Committee for Scientific Research under grant No. 8 T07A 04620.

\section{REFERENCES}

[1] Gadaj S.P., Nowacki W.K. and Pieczyska E., Changes of temperature during the simple shear test of stainless steel, Arch. of Mechanics, 48, 4, 1996, 779-788.

[2] Gary G., Nowacki W.K., Essai de cisaillement plan dynamique appliquée a des tôles minces, Journal de Physique IV, C8, 1994, 65-70.

[3] Klepaczko J.R., Nguyen H.V. and Nowacki W.K., Quasi-static and dynamic shearing of sheet metals. European Journal of Mechanics, A/Solids 18, 1999, 271-289.

[4] Oliferuk W., Kruszka L. and Nowacki W.K. Measurements of temperature during dynamic shear deformation of carbon steel. Journal de Physique 10, 9, 2000, 243 - 248.

[5] Nguyen, H.V., Nowacki, W.K., Simple shear of metal sheets at high rates of strain. Archives of Mechanics, 49, 1997, 369-384.

[6] Guélin, P., Nowacki, W.K., Nguyen, H.V., Localisation of thermo-elastoplastic deformations in the case of simple shear, Journal de Physique, 7, C3, 1997, 717-722. 


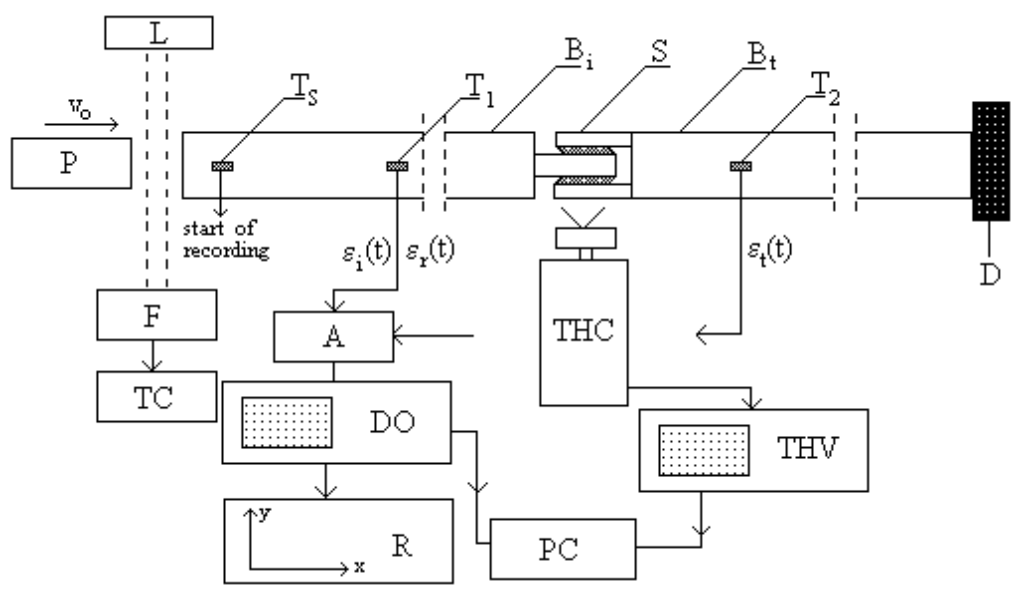

Fig. 1. Standard SHPB apparatus.

$\begin{array}{ll}P & \text { - projectile } \\ B i & \text { - incident bar } \\ B t & \text { - transmitting bar } \\ S & \text { - shear device with double shear } \\ & \text { specimen } \\ D & \text { - damper } \\ L & \text { - source of light } \\ F & \text { - photodiodes }\end{array}$

TC - time counter

$T 1, T 2$ and Ts - strain gauges

$A$ - amplifier

DO - digital oscilloscope

$P C$ - microcomputer

$R$ - xy recorder or graphic printer

THC - thermography camera

THV - oscilloscope of thermography set
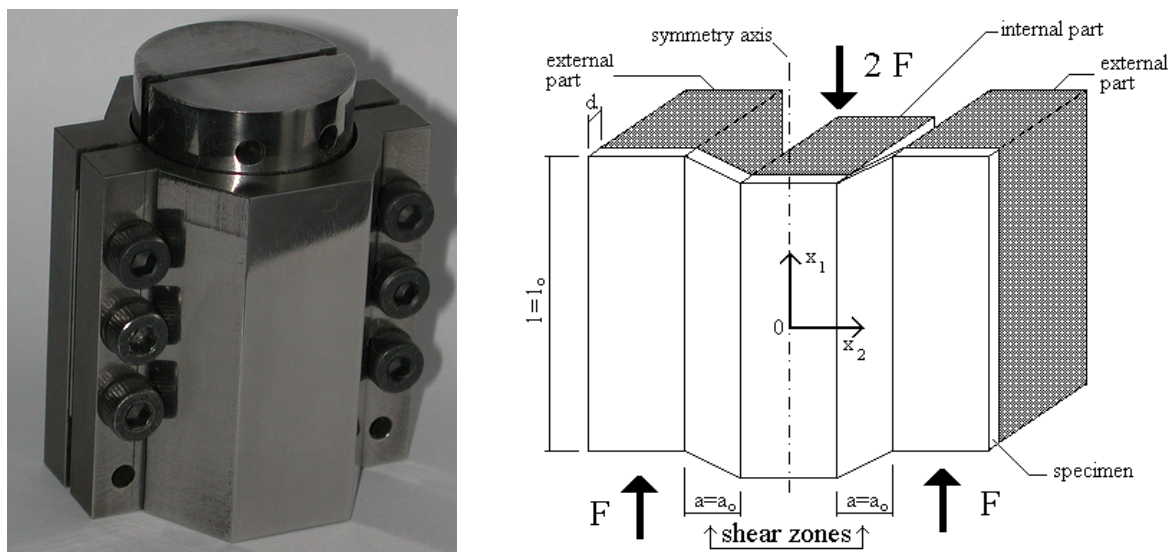

Fig. 2. Shear device; scheme of shear testing 


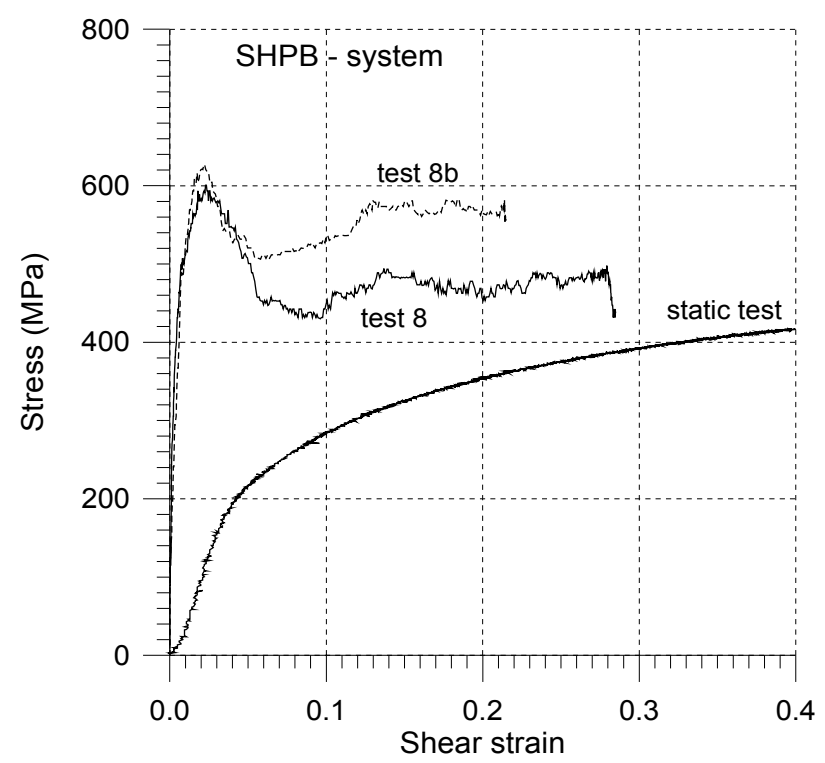

Fig. 3. Stress-strain curves during dynamic simple shear (test 8 and test $8 b$ ) - SHPB and static test; dynamic tests - strain rate $10^{3} \mathrm{~s}^{-1}$, static test - strain rate $10^{-3} \mathrm{~s}^{-1}$

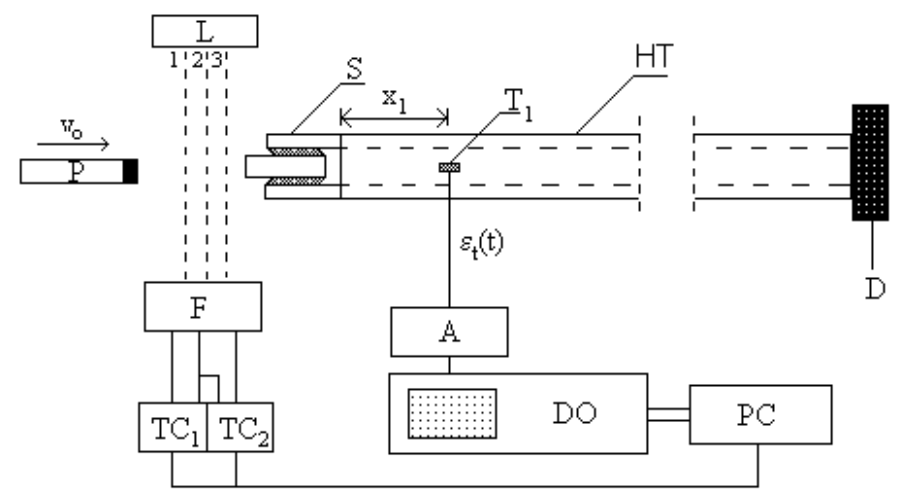

Fig. 4. Configuration of experimental set-up for direct impacts.

$S$ - shear device with a double shear specimen

HT - Hopkinson tube (transmitter bar)

$P$ - projectile

$D$ - damper

$L \quad$ - source of light

$F$ - photodiodes
TC - time counter $T_{1}$ - strain gauge

A - amplifier

$D O$ - digital oscilloscope

A - amplifier

$D O$ - digital oscilloscope

$P C$ - microcomputer 


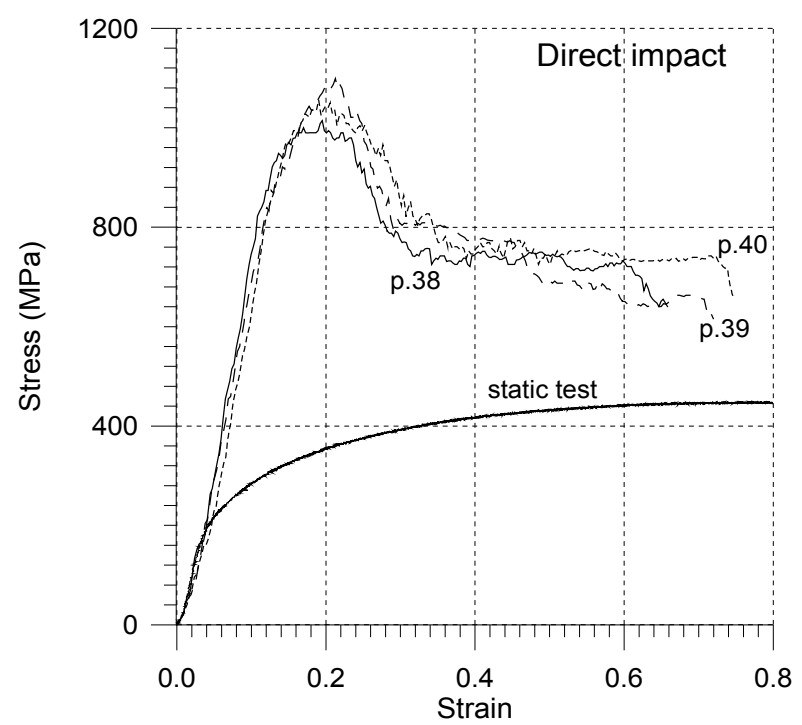

Fig. 5. Stress-strain curves during dynamic simple shear- direct impact (p38, p39, p40) and static test; dynamic tests - strain rate $8.3 \times 10^{3} \mathrm{~s}^{-1}$, static test - strain rate $10^{-3} \mathrm{~s}^{-1}$

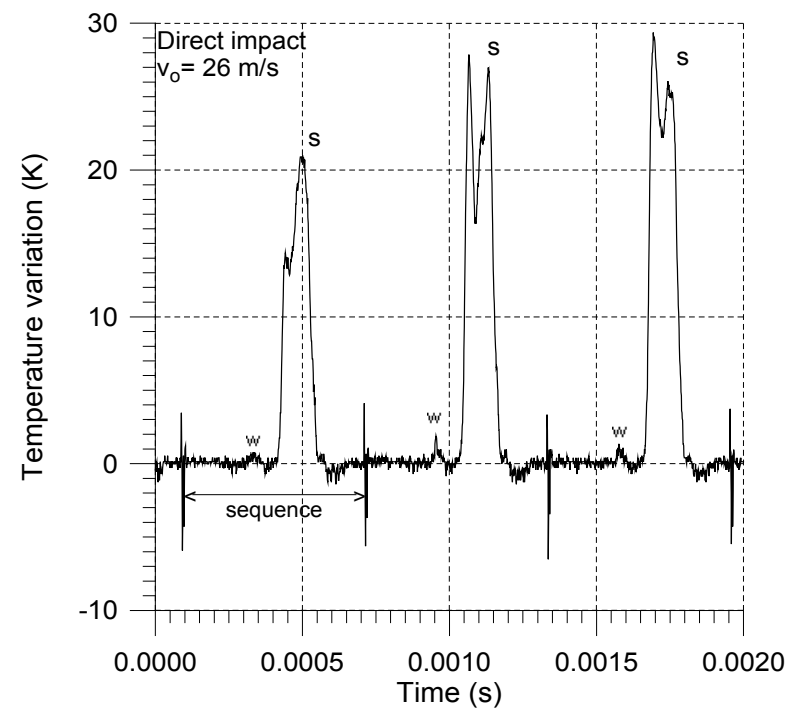

Fig. 6. Typical voltage signals (s) from the shear zone and from the indicator $(w)$ in sequential time intervals 
http://dx.doi.org/10.21611/qirt.2002.024

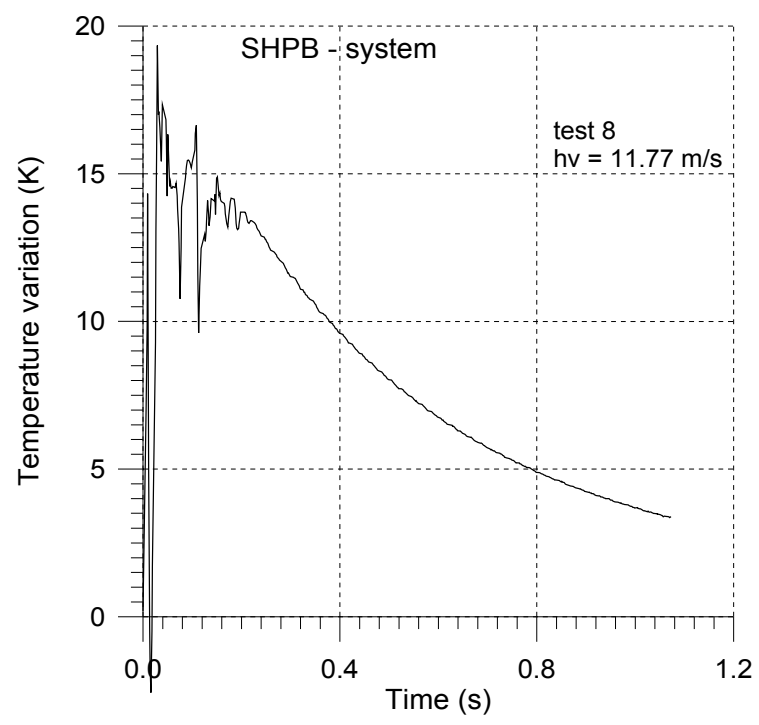

Fig. 7. Temperature vs. time obtained during dynamic simple shear (SHPB) measured by a thermography camera with $750 \mathrm{~s}^{-1}$ image rate 